\title{
Statistical visualization of the Earth's magnetotail based on Geotail data and the implied substorm model
}

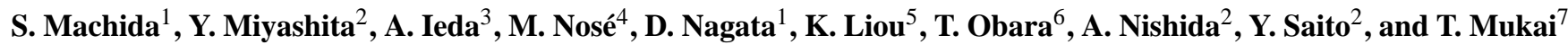 \\ ${ }^{1}$ Department of Geophysics, Graduate School of Science, Kyoto University, Kyoto, Japan \\ ${ }^{2}$ Institute of Space and Astronautical Science/JAXA, Kanagawa, Japan \\ ${ }^{3}$ Solar Terrestrial Environment Laboratory, Nagoya University, Aichi, Japan \\ ${ }^{4}$ World Data Center for Geomagnetism, Kyoto University, Kyoto, Japan \\ ${ }^{5}$ The Johns Hopkins University, Applied Physics Laboratory, MD, USA \\ ${ }^{6}$ Aerospace Research and Development Directorate/JAXA, Ibaraki, Japan \\ ${ }^{7}$ Japan Aerospace Exploration Agency (JAXA), Tokyo, Japan
}

Received: 16 April 2008 - Revised: 18 November 2008 - Accepted: 17 December 2008 - Published: 2 March 2009

\begin{abstract}
We investigated the temporal and spatial development of the near-Earth magnetotail during substorms based on multi-dimensional superposed-epoch analysis of Geotail data. The start time of the auroral break-up $(t=0)$ of each substorm was determined from auroral data obtained by the Polar and IMAGE spacecraft. The key parameters derived from the plasma, magnetic-field, and electric-field data from Geotail were sorted by their meridional $X$ (GSM) $Z$ (proxy) coordinates.

The results show that the Poynting flux toward the plasmasheet center starts at least $10 \mathrm{~min}$ before the substorm onset, and is further enhanced at $X \sim-12 R_{E}$ (Earth radii) around 4 min before the onset. Simultaneously, large-amplitude fluctuations occurred, and earthward flows in the central plasma sheet between $X \sim-11 R_{E}$ and $X \sim-19 R_{E}$ and a duskward flow around $X=-10 R_{E}$ were enhanced. The total pressure starts to decrease around $X=-16 R_{E}$ about $4 \mathrm{~min}$ before the onset of the substorm. After the substorm onset, a notable dipolarization is observed and tailward flows commence, characterised by southward magnetic fields in the form of a plasmoid.

We confirm various observable-parameter variations based on or predicted by the relevant substorm models; however, none of these can explain our results perfectly. Therefore, we propose a catapult (slingshot) current-sheet relaxation model, in which an earthward convective flow produced by catapult current-sheet relaxation and a converted duskward flow near
\end{abstract}

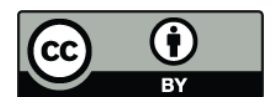

Correspondence to: $\mathrm{S}$. Machida (machida@kugi.kyoto-u.ac.jp) the Earth are enhanced through flow braking around $4 \mathrm{~min}$ before the substorm onset. These flows induce a ballooning instability or other instabilities, causing the observed current disruption. The formation of the magnetic neutral line is a natural consequence of the present model, because the relaxation of a highly stretched catapult current-sheet produces a very thin current at its tailward edge being surrounded by intense earthward and tailward magnetic fields which were formerly the off-equatorial lobe magnetic fields. This location is the boundary between a highly stressed catapult current sheet and a Harris-type current sheet characterized by little stress. In addition, the flows induced around the boundary toward the current-sheet center may enhance the formation of the magnetic neutral line and the efficiency of magnetic reconnection. After magnetic reconnection is induced, it plays a significant role in driving the substorm.

Keywords. Magnetospheric physics (Magnetotail; Storms and substorms) - Space plasma physics (Nonlinear phenomena)

\section{Introduction}

Numerous studies have sought to understand substorms (e.g. Akasofu, 1964; Russell and McPherron, 1973; Nishida, 1978); however, no consensus has been reached regarding the initial triggering mechanism.

One of the relevant substorm models, the near-Earth neutral-line (NENL) model, explains the three phases of a substorm. When the interplanetary magnetic field turns

Published by Copernicus Publications on behalf of the European Geosciences Union. 


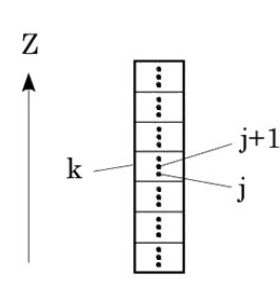

$\beta$ xi $(\mathbf{j}+1)<\beta$ xi (j)

$\beta \times \mathbf{i}(\mathbf{j})=\mathbf{n k T i} /\left(\mathbf{B x}(\mathbf{j})^{2} / 2 \mu_{0}\right)$

North-South Symmetry

$<\mathbf{Q}_{\mathrm{k}}>=\Sigma \mathbf{Q}(\mathbf{j}) / \mathbf{N}_{\mathrm{k}}$

$\mathbf{Z}$

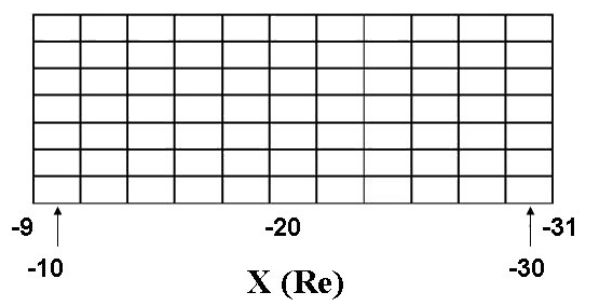

Fig. 1. Schematic illustration of the analysis method.

southward, the dayside reconnection is enhanced and the growth phase starts. The transport of magnetic flux into the tail lobe intensifies the cross-tail current and causes plasmasheet thinning during this phase. The expansion phase is induced by the formation of the NENL, and subsequent earthward flows due to magnetic reconnection transport the northward magnetic field lines, causing dipolarization and an injection of energized particles into the inner magnetosphere. In the downtail region, the fast tailward flows consist of a plasmoid. The NENL resides in the near-Earth tail until the recovery phase starts. The plasma sheet is thickened in accordance with the neutral line's retreat to the downtail region in the recovery phase (Hones, 1976; Baker et al., 1996).

On the other hand, the current-disruption model (Chao et al., 1977; Lui et al., 1990; Lui, 1996) predicts that the disruption of the cross-tail current begins at a distance of $\sim 8 R_{E}$ (Earth radii) in the pre-midnight magnetosphere and the current starts to flow into the ionosphere, thus initiating the substorm. The disruption of the current can account for particle injection and energizing by convection surges of the inner magnetosphere. A rarefaction wave is launched from the current-disruption region, accompanying the plasma-sheet thinning and southward dipping of the magnetic field in the mid-tail region. Magnetic reconnection starts when this rarefaction wave propagates down to $X \sim-20 R_{E}$ and induces the formation of the magnetic neutral line.

In a similar scenario to this latter model, a ballooninginstability model has been proposed, in which a ballooning instability (Roux et al., 1991; Miura et al., 1989; Cheng and Lui, 1998) is responsible for the explosive conversion of magnetic-field energy into the kinetic energy of the particles. The excited surface waves propagate westward with the drifting ions. This disturbed region in the equatorial magnetosphere can be mapped to the ionosphere as a form of the westward-traveling surge.
In a model that we tentatively call the plasma-sheet divergence model, a reduction of plasma convection is predicted, which follows a growth-phase period of enhanced convection, and causes a divergence of plasma-sheet particles driven by diamagnetic drift and leading to a flux-tube content reduction. This region is expected to be the current-disruption region, and the substorm is triggered by the reduction in plasma convection (Lyons et al., 2003).

Another relevant substorm model is the thermal catastrophe model (Smith et al., 1986), in which Alfvénic turbulent waves propagate toward the central plasma sheet (CPS). Their subsequent conversion into kinetic Alfvén waves and absorption of the wave energy by particles induces catastrophic changes in the plasma-sheet conditions, resulting in substorms. In another possible model, a Kelvin-Helmholtz instability grows between the tailward magnetosheath and earthward flows in the plasma-sheet boundary layer (PSBL) on the flank of the magnetotail, contributing to the onset of the substorm. This model is called the boundary-layer model (Rostoker and Eastman, 1987).

In the magnetosphere-ionosphere (M-I) coupling model (Kan et al., 1988), the substorm is triggered by upward field-aligned current enhancements due to M-I coupling in the midnight sector near the poleward boundary of a highconductance belt associated with diffuse auroral precipitation. The necessary conditions in this model are that the polar-cap potential must exceed a certain value (about $70 \mathrm{kV}$ ) and the convection-reversal region must overlap with the poleward gradient of the diffuse auroral conductance in the ionosphere in the midnight sector. The substorm subsides when one of these two necessary conditions is no longer satisfied.

To assess the validity of these models, we performed a statistical study using Geotail data, one of the best available datasets to date for the study of the magnetotail in the region covering $-10>X\left(R_{E}\right)>-30$.

\section{Method of analysis}

The Geotail data employed in this study include ion-velocitymoment data from the Low Energy Particle instrument (LEP) (Mukai et al., 1994), magnetic-field data from the Magnetic Field instrument (MGF) (Kokubun et al., 1994), and electricfield data from the Electric Field Detector (EFD) (Tsuruda et al., 1994), all with a time resolution of $12 \mathrm{~s}$. To determine the onset time of substorms with auroral break-up, we used the data from the Ultraviolet Imager (UVI) (Torr et al., 1995) and the Far Ultraviolet Imager (FUV) (Mende et al., 2000) on the Polar and IMAGE spacecraft, respectively.

We selected events detected when Geotail was located in the region $-9>X\left(R_{E}\right)>-31$ and $-3<Y\left(R_{E}\right)<8$, in GSM (Geocentric Solar Magnetospheric) coordinates, and distributed them evenly into columns between $-10 R_{E}$ and $-30 R_{E}$, with a width of $2 R_{E}$ (see Fig. 1). The numbers 

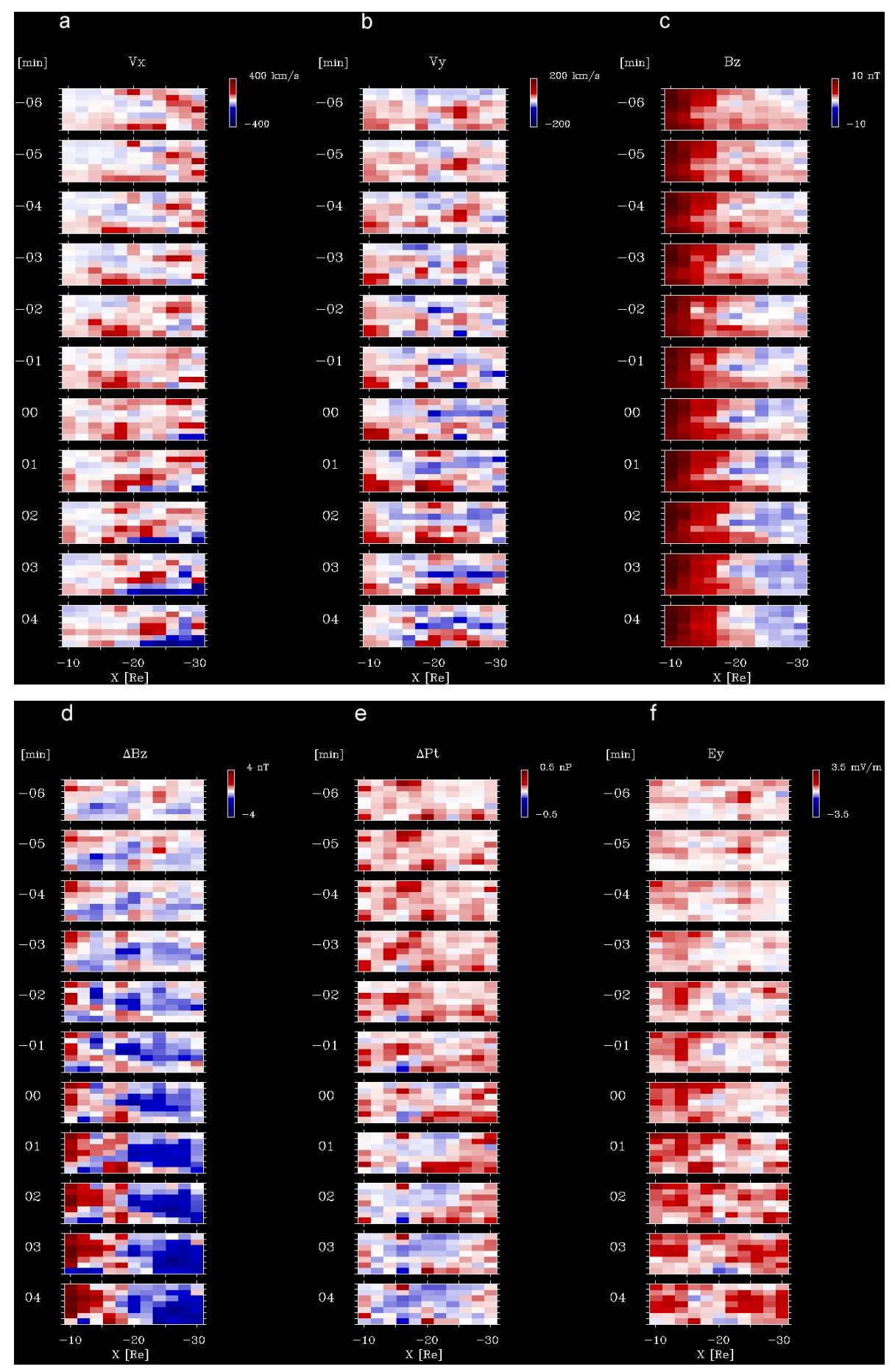

Fig. 2. Results of the analysis: (a) earthward flow velocity $\left(V_{x}\right)$, (b) duskward flow velocity $\left(V_{y}\right)$, (c) northward magnetic field $\left(B_{z}\right)$, (d) deviation of the northward magnetic field $\left(\Delta B_{z}\right)$, (e) deviation of the total pressure $\left(\Delta P_{t}\right)$, (f) duskward electric field $\left(E_{y}\right)$, (g) DC Poynting flux toward plasma-sheet center $\left(F_{\text {poyz }}\right)$, (h) rms value of the magnetic field $\left(B_{\mathrm{rms}}\right)$, (i) rms value of the electric field $\left(E_{\mathrm{rms}}\right)$.

of events assigned to the different columns are $30,28,16$, $12,14,13,16,16,25,22$, and 42 , from the earthward to the tailward direction; i.e., from $X=-9 R_{E}$ to $-31 R_{E}$. Thus, the total number of events considered is 234 . We set the time window at 2 min, so that each event consists of 10 datasets because of the sampling time of $12 \mathrm{~s}$. Approximately 2300 


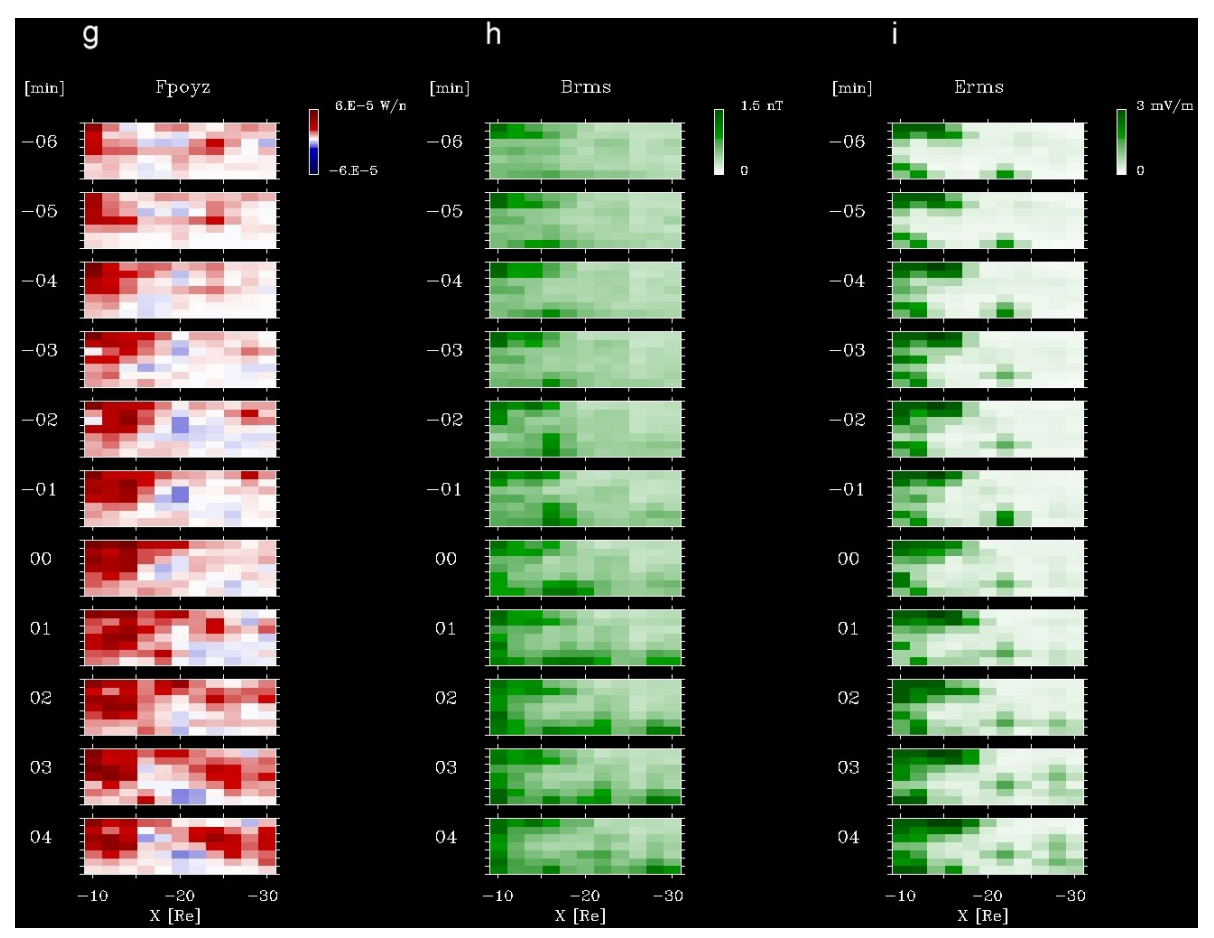

Fig. 2. Continued.

datasets were used to infer the spatial structure in specific 2 min intervals.

In each column, we sorted the data assuming that events with higher values of the parameter $\beta_{i x}=n k T_{i} /\left(B_{x}^{2} / 2 \mu_{0}\right)$ were located nearer the plasma-sheet center, where $n, T_{i}, B_{x}, k$, and $\mu_{0}$ are the ion number density and temperature, the $X$ component of the magnetic field, Boltzmann's constant, and the magnetic permeability of free space, respectively. Next, we separated the data into seven bins aligned along the GSM $Z$ direction for each column. We then obtained the average values for various parameters to determine the spatial structure in different time intervals. We assumed north-south symmetry and combined the data in the northern and southern magnetotails, regardless of the sign of $B_{x}$.

In an ordinary superposed-epoch analysis, a given parameter is plotted as a function of time, starting from a specific common event at $t=0$. In contrast, we show variations for a given parameter in the two-dimensional $X(\mathrm{GSM})-Z$ (proxy) plane as a function of time. One parameter is shown as a function of three parameters: $X(\mathrm{GSM}), Z$ (proxy), and $t$. For this reason, we refer to our method as a multi-dimensional superposed-epoch analysis.

An advantage of our method compared with those that take $\beta_{i x}$ as the ordinate is that it provides information regarding the scale length (thickness) in the $Z$ direction, since the probability of encountering a particular structure is approximately proportional to its thickness.

\section{Results}

We performed a series of superposed-epoch analyses using Geotail data to determine which model can explain the actual substorms that occur in the real world. Our previous studies (Machida et al., 1999; Miyashita et al., 1999, 2000, 2003) mainly focused on substorm structural changes in the $X-Y$ plane (i.e. the equatorial projection of the near-Earth magnetotail). Recently, a further study was performed by Miyashita et al. (2009) following this concept. In this study, we obtained variations in the meridional $X-Z$ plane based on multi-dimensional superposed-epoch analysis (Machida et al., 2000), and found further detailed variations that complement our previous results.

Figure 2a shows the earthward flow velocity, $V_{x}$, consisting of both parallel and perpendicular components with respect to the magnetic field. The earthward flow in the PSBL, possibly originating from the distant neutral line (DNL), had already been present (Rostoker and Eastman, 1987) from $t=-6 \mathrm{~min}$, which corresponds to the late substorm-growth phase. The presence of an earthward PSBL flow is consistent with both the boundary-layer (Rostoker and Eastman, 1987) and the NENL models (Hones, 1976; Baker et al., 1996). There exists another earthward flow in the CPS, between $X=-15 R_{E}$ and $-25 R_{E}$, which is essentially a busty bulk flow (Baumjohann et al., 1990; Angelopoulos et al., 1992; Slavin et al., 1997). The location of the intense flow moves earthward of $X \sim-20 R_{E}$ at $t=-4 \mathrm{~min}$ and tailward 
after the onset of the substorm. The velocity of the earthward flow reduces inside of $X \sim-13 R_{E}$. This corresponds to flow braking (Shiokawa et al., 1997) caused by the encounter of the fast flow with the high-pressure region on the near-Earth side; the plasma starts to flow around the dawn and dusk sides at this location, as will be shown below.

A notable tailward flow in the downtail at $X=-20 R_{E}$ starts at $t=0$, consistent with a recent tailward-flow study (Ieda et al., 2008). This tailward flow grows into a welldeveloped plasmoid (Birn and Hesse, 1991; Machida et al., 1994; Ieda et al., 1998) in the downtail region.

The NENL is located around $X=-20 R_{E}$, the exact position of which may differ somewhat from event to event. As a result, the earthward flows can be observed at $X=-20 R_{E}$ when the NENL is formed in the tailward of $X=-20 R_{E}$, and the tailward flows can be observed when the NENL is formed in the earthward of $X=-20 R_{E}$. Therefore the latitudinal structure appearing in $V_{x}$ plot of Fig. 2a has to be considered carefully. Namely, the simultaneous appearance of tailward flows in the plasma sheet center and earthward flows in the higher latitude at around $X=-22 R_{E}$ after $t=1 \mathrm{~min}$ is not a real picture of single substorm event. We think that this reflects that the values of $\beta_{i x}$ for the tailward flows are higher than those for the earthward flows.

The earthward flows occurring before the substorm onset are anticipated by the NENL model; however, the tailward flows develop after $t=0$, which is in conflict with the predictions from the NENL model. Instead, tailward flows are expected to develop a few minutes earlier than the substorm onset, at the same time as the earthward flows.

Also, the location of earthward CPS flows advances earthward before the onset, and it retreats tailward after the onset. This character has not been predicted by any existing model of substorm.

Figure $2 \mathrm{~b}$ shows the duskward flow velocity, $V_{y}$. In this study, we restricted the region in the dawn-to-dusk direction to $-3<Y\left(R_{E}\right)<8$. Therefore, duskward flows dominate the dawnward flows in the region $-9>X\left(R_{E}\right)>-13$ (see Fig. 2b), consistent with the result obtained by Paterson et al. (1998). This flow is enhanced at $t=-3 \mathrm{~min}$ and quenched at $t=4 \mathrm{~min}$. It seems that this duskward flow is an extension of the earthward flow in the CPS, turning its flow vector duskward by means of a flow-braking process. These flows appear to be closely related to the substorm onset. Before the onset, relatively intense duskward flows can also be found on the earthward and plasma-sheet sides of the PSBL flows in the region $-21>X\left(R_{E}\right)>-27$.

Intense duskward flows are also present around $X=-18 R_{E}$ from $t=-6 \mathrm{~min}$. After $t=1 \mathrm{~min}$, this region expands tailward, and the duskward flow in the current sheet connects to both the earthward and tailward components of the NENL. Dawnward flows also appear on the lobeside of the downtail at $X \sim-17 R_{E}$. This can be a cold-ion drift, and is found near the separatrix of the magnetic reconnection topology (e.g. Nakabayashi and Machida, 1997; Fujimoto and Machida, 2007).

The variation of the northward magnetic field, $B_{z}$, is shown Fig. 2c. The value of $B_{z}$ is large in the near-Earth region due to the contribution from the Earth's dipole field. The other notable feature is that $B_{z}$ becomes negative in the off-equatorial region at $X<-17 R_{E}$; the region of negative $B_{z}$ expands over time. To quantify the variation in $B_{z}$, we obtained $\Delta B_{z}$, i.e. the deviation of the northward magnetic-field component from the average value in the time interval from $t=-12$ to $t=-10 \mathrm{~min}, \Delta B_{z}=<B_{z}(t-1$ to $t+1 \mathrm{~min})>-<B_{z}$ $(-12$ to $-10 \mathrm{~min})>$. The result is shown in Fig. 2d. A notable characteristic of this panel is that the region represented in red, which corresponds to an increase in the northward magnetic field, first appears on the lobeside at $X=-10 R_{E}$, and this variation expands toward the plasma-sheet center as well as in the tailward direction as time progresses. The increase in $B_{z}$ well before $t=0$ on the earthward side is thought to be due to the accumulation of magnetic-field lines convected from the lobeside, and the increase around and after $t=0$ is related to dipolarization (Nagai, 1982). Other groups of $B_{z}$ increases are associated with earthward CPS flows between $X=-13 R_{E}$ and $X=-20 R_{E}$ (Shue et al., 2008). At the same time, regions characterized by negative $\Delta B_{z}$ values, showing a southward increase in the magnetic field, are also present, exhibiting two traces extending from the lower-left to the upper-right direction in the region between $X=-11 R_{E}$ and $X=-21 R_{E}$ and from $X=-25 R_{E}$ to $X=-31 R_{E}$. The location of the upper-right edge of the former expands toward the plasma-sheet center and merges with the latter at $t=-3 \mathrm{~min}$. The southward increase in the magnetic field further develops over time in the region tailward of $X=-20 R_{E}$. This region correlates with the initial plasmoid position and its surrounding regions. Those variations are compatible with the predictions of the NENL (Hones, 1976; Baker et al., 1996) and the current-disruption models (Chao et al., 1977; Lui et al., 1990; Lui, 1996).

Figure $2 \mathrm{e}$ shows the deviation of the total pressure (i.e. the sum of the plasma and magnetic pressures) from the average value from $t=-12$ to $-10 \mathrm{~min}, \Delta P_{t}=<P_{t}(t-$ 1 to $t+1 \mathrm{~min})>-<P_{t}(-12$ to $-10 \mathrm{~min})>$. The total pressure enhancement first appears in the lobe region at $-15>X\left(R_{E}\right)>-19$ and in the CPS at $-20>X\left(R_{E}\right)>-28$. The region of total pressure decrease (i.e. the rarefaction region) first seems to appear in the plasma sheet around $X=-16 R_{E}$ at $t=-4 \mathrm{~min}$, and then propagates both earthward and tailward along the lobes, consistent with the recent result obtained by Miyashita et al. (2009). It is not necessarily clear, but magnetic reconnection seems to occur when the rarefaction region reaches $X \sim-20 R_{E}$. This resembles the variation predicted by the current-disruption model (Chao et al., 1977; Lui et al., 1990; Lui, 1996), although the rarefaction region starts around $X=-8 R_{E}$ in this model and it propagates down to $X \sim-20 R_{E}$, where it proceeds to trigger the magnetic reconnection. 
The variation in the duskward electric field, $E_{y}$, is shown in Fig. 2f. Some enhancement of this parameter can be found in the upper-left corner (i.e. in the earthward lobe at $X \sim-10 R_{E}$ ) and in the region around $X=-24 R_{E}$ at $t=-6 \mathrm{~min}$. The former variation is further enhanced at $X \sim-12 R_{E}$ at $t=-4 \mathrm{~min}$, and the enhancement expands tailward and also to the plasma-sheet center, while the latter variation seems to be quenched once just before the substorm onset. Notable enhancements appear tailward of $X \sim-20 R_{E}$ just after the onset, which is thought to be the result of magnetic reconnection. However, based on the NENL model (Hones, 1976; Baker et al., 1996), we expect an enhancement of the duskward electric field before the substorm onset, possibly at $t=-4 \mathrm{~min}$ at $X \sim-20 R_{E}$. This characteristic is not necessarily evident in our data.

If we compare Fig. $2 \mathrm{f}$ with Fig. 2a, we find that the region of the duskward electric field at $t=-6 \mathrm{~min}$ at high latitudes near $X=-23 R_{E}$ is located just below the PSBL flow from the DNL. The exact mechanism remains unclear, but observations suggest that the plasma is convected toward the plasma-sheet center. At the same time, the plasma located earthward of that position drifts upward (away from the plasma-sheet center), where we find a weak dawnward electric field from $t=-3 \mathrm{~min}$ to $t=-1 \mathrm{~min}$, corresponding to this drift. In the region between $X=-9 R_{E}$ and $-15 R_{E}$, we also find a strong duskward electric field, which may be related to both the penetration of the Poynting flux from the lobeside and the dipolarization. After $t=0$, the duskward electric field is widely enhanced downtail of $X=-20 R_{E}$, which is related to magnetic reconnection. Interestingly, the duskward electric field around $X=-20 R_{E}$ is not enhanced, even after $t=0$, when magnetic reconnection starts and the NENL is formed around this region.

Figure $2 \mathrm{~g}$ shows the Poynting flux of the direct-current (DC) component, using $1 / 12 \mathrm{~Hz}$ cut-off frequency electric and magnetic fields toward the plasma-sheet center, $F_{\text {poyz }}$, the direction of which is vertically downward in each panel. There is a clear enhancement of this parameter around $X \sim-10 R_{E}$ at $t=-6 \mathrm{~min}$, from which the region of enhanced $F_{\text {poyz }}$ propagates tailward. A notable enhancement around $X=-12 R_{E}$ at $t=-4 \mathrm{~min}$ was also found, related to the intensification of $E_{y}$. A comparison of Fig. $2 \mathrm{~g}$ with Fig. 2d reveals that the $\Delta B_{z}<0$ region is located on the CPS side and downtail of the enhanced- $F_{\text {poyz }}$ region. The negative variation in $B_{z}$ is caused by magnetic-field bending toward the plasma-sheet center associated with the enhancement of the DC Poynting flux or, equivalently, plasma convection toward the plasma-sheet center. This signature is also anticipated as a variation associated with plasma-sheet thinning (Akasofu, 1977; Lui et al., 1977), which is predicted by both the NENL (Hones, 1976; Baker et al., 1996) and current-disruption models (Chao et al., 1977; Lui et al., 1990; Lui, 1996). The Poynting-flux enhancement is, in a sense, analogous to the necessary conditions for the thermalcatastrophe model (Smith et al., 1986); Alfvénic alternating- current waves are assumed in that model, but the DC component is plotted in Fig. 2g. The enhancement of $F_{\text {poyz }}$ after $t=0$ in the $X<-20 R_{E}$ region is caused by magnetic reconnection.

Figure $2 \mathrm{~h}$ shows $B_{\mathrm{rms}}$, the root-mean-square (rms) value of the magnetic-field deviation from the average value using $1 / 16 \mathrm{~s}$ sampling once every $12 \mathrm{~s}$. This parameter shows the magnetic-field intensity of low-frequency electromagnetic waves in the 0.17 to $8 \mathrm{~Hz}$ range. At $t=-6 \mathrm{~min}$, this parameter is enhanced in two distinct regions in the nearEarth zone, at $-10>X\left(R_{E}\right)>-16$. One of these regions is located on the lobeside and the other near the plasma-sheet center. Intense wave activity on the lobeside is related to the enhanced Poynting flux toward the plasma-sheet center. On the other hand, the enhanced Poynting flux in the corresponding region located near the CPS is related to thermal fluctuations in the high-beta plasma sheet and also to the excitation of unstable waves associated with either an intensification of the cross-tail current or a high-speed flow. The large $B_{\text {rms }}$ region near the plasma-sheet center is centered at $X \sim-16 R_{E}$ at $t=-4 \mathrm{~min}$. This active region extends to $X=-21 R_{E}$ at $t=0$ and extends further down to $X=-31 R_{E}$ at $t=1 \mathrm{~min}$. Beyond $X=-21 R_{E}$, large electromagnetic fluctuations are only found in the CPS region, likely associated with the tailward flow of the plasmoid. Thus, the region characterised by large electromagnetic fluctuations extends from the earthward side to the downtail region as time progresses. Although the location of the intense waves is different from the original proposal, the wave-turbulence character resembles the situation predicted by the current-disruption model (Lui et al., 2006).

A similar rms value of the electric-field variations from the average value with $1 / 32 \mathrm{~s}$ sampling for every $12 \mathrm{~s}$, $E_{\text {rms }}$, is shown in Fig. 2i. Again, this parameter represents the electric-field intensity of the low-frequency waves. There exist four distinct regions of strong wave intensity: the near-Earth lobe at $-9>X\left(R_{E}\right)>-18$, the near-Earth plasma-sheet center at $-9>X\left(R_{E}\right)>-15$, the region around $X \sim-22 R_{E}$, and the plasma sheet at $-25>X\left(R_{E}\right)>-31$. The third region is close to the location where the NENL is formed. Further, the waves found in the fourth region are less intense and enhanced after the onset, and they seem to be related to the tailward flows produced by magnetic reconnection.

To characterise the variations in the different regions more quantitatively, we calculated several key parameters that characterise the magnetotail variations immediately before and after the substorm onset (see Fig. 3). Figure 3a shows the time variations of $F_{\text {poyz }}$ in region I $\left(-9>X\left(R_{E}\right)>-19\right.$; $4<Z / \Delta Z<7)$ and $\Delta P_{t}$ in region IV $\left(-13>X\left(R_{E}\right)>-17\right.$; $0<Z / \Delta Z<2$ ), the locations of which are defined in Fig. 4 . Here, $\Delta Z$ is the spatial width of the bin in the $Z$ direction, which is about $0.86 R_{E}$ because the $Z$ coordinate of the Geotail locations in the GSM coordinate system is distributed throughout the range from approximately $-6 R_{E}$ to $+6 R_{E}$, according to the Tsyganenko and Fairfield model 

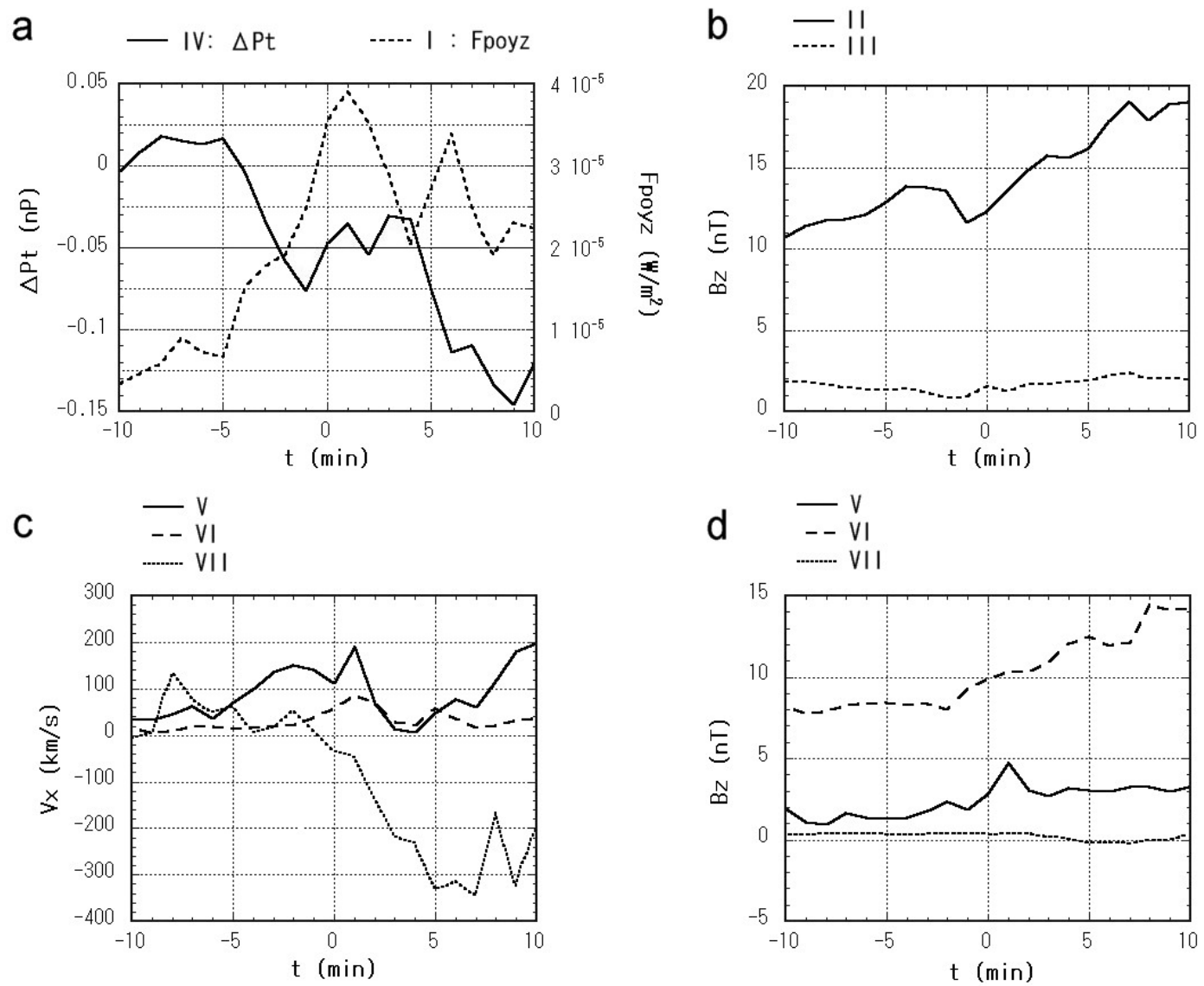

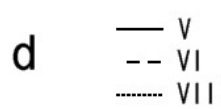

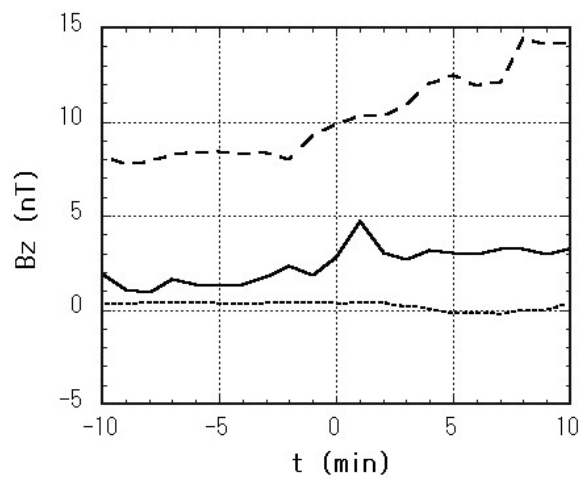

Fig. 3. Time variations of the relevant parameters: (a) $\Delta P_{t}$ in region IV and $F_{\text {poyz }}$ in region I, (b) $B_{z}$ in regions II and III, (c) $V_{x}$ in regions V, VI, and VII, (d) $B_{z}$ in regions V, VI, and VII.

(Tsyganenko and Fairfield, 2004).

There is already an increase in Poynting flux toward the plasma-sheet center at $t=-10 \mathrm{~min}$, but it is enhanced at $t=-4 \mathrm{~min}$. In contrast, the total pressure, $P_{t}$, starts to decrease at $t=-4 \mathrm{~min}$, although there is a slight increase from $t=-1 \mathrm{~min}$ to $5 \mathrm{~min}$. Both curves show an approximate anticorrelation with each other.

Figure $3 b$ shows the time variations of the northward magnetic-field component, $B_{z}$, in region II $\left(-9>X\left(R_{E}\right)>-11 ; 4<Z / \Delta Z<7\right)$ and region III $\left(-13>X\left(R_{E}\right)>-21 ; 2<Z / \Delta Z<6\right)$ of Fig. $4 . \quad$ As it is associated with the enhancement of $F_{\text {poyz }}, B_{z}$ increases due to the accumulation of the magnetic fields in region II throughout the time interval shown in Fig. 3b, except around $t=-1 \mathrm{~min}$ where a decrease is seen. On the other hand, the decrease due to magnetic-field bending can be found at the beginning, and continues until $t=-1 \mathrm{~min}$, from which time $B_{z}$ increases in region III.

The temporal variations in earthward flow velocity, $V_{x}$, in regions $\mathrm{V}\left(-15>X\left(R_{E}\right)>-19 ; 0<Z / \Delta Z<2\right)$, VI $\quad\left(-9>X\left(R_{E}\right)>-11 ; \quad 0<Z / \Delta Z<4\right), \quad$ and $\mathrm{VII}$ $\left(-21>X\left(R_{E}\right)>-29 ; 0<Z / \Delta Z<1\right)$ are shown in Fig. 3c.

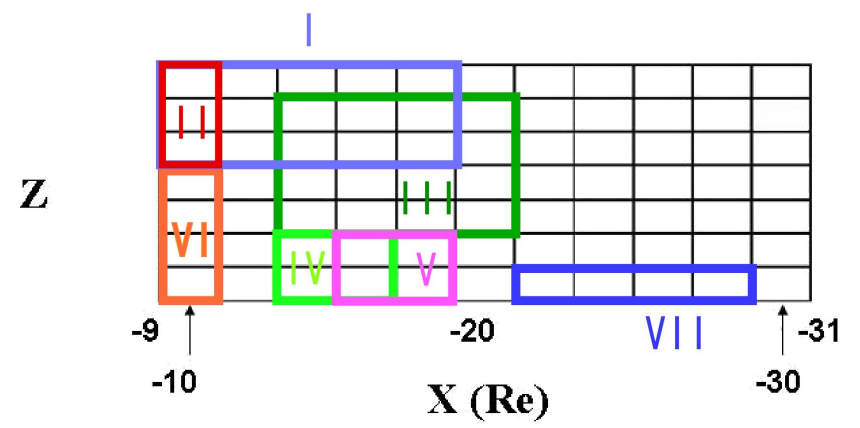

Fig. 4. Definition of the selected regions shown in Fig. 3.

There exists an enhancement of the earthward flows in region $\mathrm{V}$ from around $t=-5 \mathrm{~min}$, with a peak of $190 \mathrm{~km} \mathrm{~s}^{-1}$ at $t=1 \mathrm{~min}$; they then quench for short time intervals until they start recovering from $t=4 \mathrm{~min}$. The earthward flows in region VI seem to be enhanced at $t=2 \mathrm{~min}$, with a peak of $80 \mathrm{~km} \mathrm{~s}^{-1}$ at $t=2 \mathrm{~min}$. The variation in $V_{x}$ in region VII indicates a slow earthward flow until $t=-1 \mathrm{~min}$, upon which it changes into a tailward flow with a maximum flow speed of $-340 \mathrm{~km} \mathrm{~s}^{-1}$ at $t=7 \mathrm{~min}$. 


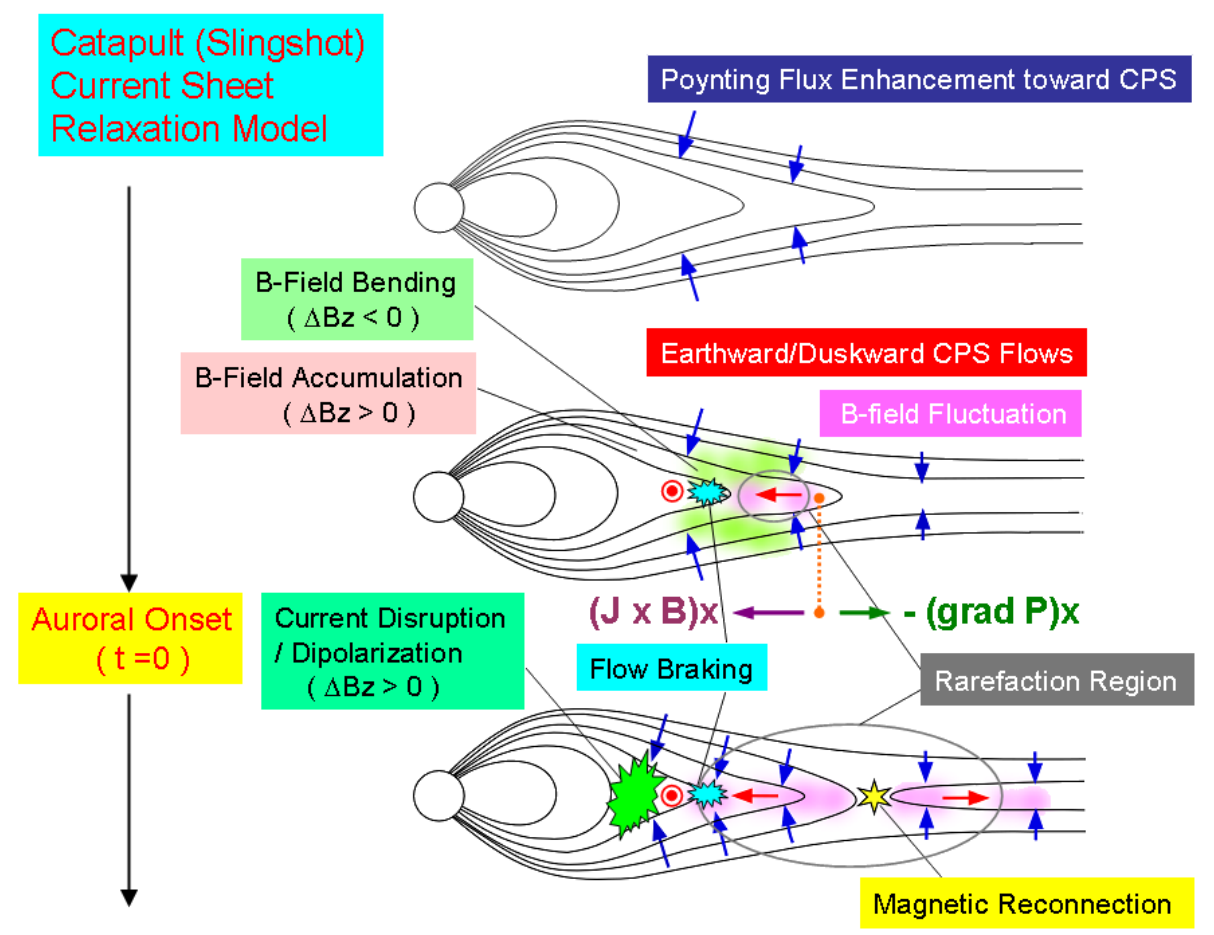

Fig. 5. Conceptual illustration of the catapult (slingshot) current-sheet relaxation model.

The variations in $B_{z}$ in the same regions (i.e. V, VI, and VII) are shown in Fig. 3d. The gradual increase of $B_{z}$ in region $\mathrm{V}$ seems to start at $t=-4 \mathrm{~min}$, with a spiky peak at $t=1 \mathrm{~min}$. A rapid increase in $B_{z}$, most likely related to dipolarization, is seen in the curve of region VI. In contrast, the value of $B_{z}$ in region VII starts to decrease at $t=2 \mathrm{~min}$ and it becomes negative (i.e. the magnetic-field direction turns southward for a short time from $4 \mathrm{~min}$ to $7 \mathrm{~min}$ after the substorm onset), after which it recovers. We believe that the tailward flow starting at $t=-1 \mathrm{~min}$ is created by magnetic reconnection, and that it accompanies the southward magnetic field $\left(B_{z}<0\right)$, but the magnitude of $B_{z}$ is minus several $\mathrm{nT}$, the effect of which is smeared out by large positive- $B_{z}$ fields associated with the pre-existing plasma sheet, until the region is predominantly occupied by tailward flows due to magnetic reconnection.

The boundary inside and outside of which the duskward and earthward plasma flows dominate is located around $X=-13 R_{E}$. As explained above, we relate this boundary to flow braking. It is also possible that these flows are related to the flows that play a significant role in the plasma-sheet divergence model. The total pressure decrease, which is similar to the flux-tube content reduction predicted by that model, first occurs at $X \sim-16 R_{E}$ in our data. This location seems to be slightly tailward compared with the location expected based on the plasma-sheet divergence model, which predicts that the reduction in plasma convection and subsequent fluxtube content reduction occur in the current-disruption region, generally considered to be at $X \sim-8 R_{E}$.
We used the time when auroral break-up occurred as a time mark in applying our multi-dimensional superposed-epoch analysis. Evidently, auroral break-up is directly related to enhancements of the M-I coupling. Both our study and the M-I coupling model (Kan et al., 1988) share this notable variation in the aurora at the time of the substorm onset; however, if enhancements of the M-I coupling indeed trigger substorms, other distinct variations (e.g. the dipolarization or magnetic reconnection) must be delayed relative to the time of auroral break-up. Such a signature is different from our result, in which those three variations occur almost simultaneously.

\section{Discussion}

We confirmed the evidence or events predicted by each of the substorm models considered. By scrutinizing our results, we found that an enhancement of the Poynting flux toward the plasma-sheet center starts at least 10 min before the substorm onset; it is further enhanced at $X \sim-12 R_{E}$ about $4 \mathrm{~min}$ before the onset. Simultaneously, large-amplitude magnetic fluctuations occurred, while earthward flows in the CPS between $X \sim-11 R_{E}$ and $X \sim-19 R_{E}$ and the duskward flow around $X=-10 R_{E}$ were enhanced. The total pressure starts to decrease around $X=-16 R_{E}$ at $t=-4 \mathrm{~min}$.

The enhancement in $F_{\text {poyz }}$, the earthward flow $V_{x}$ in the CPS, the duskward flow $V_{y}$ in the near-Earth region and the magnetic fluctuation $B_{\mathrm{rms}}$, and the decrease of $P_{t}$ all seem to start at $t \sim-4 \mathrm{~min}$. Therefore, it is difficult to reach a definite conclusion regarding their causal relationship; however, 


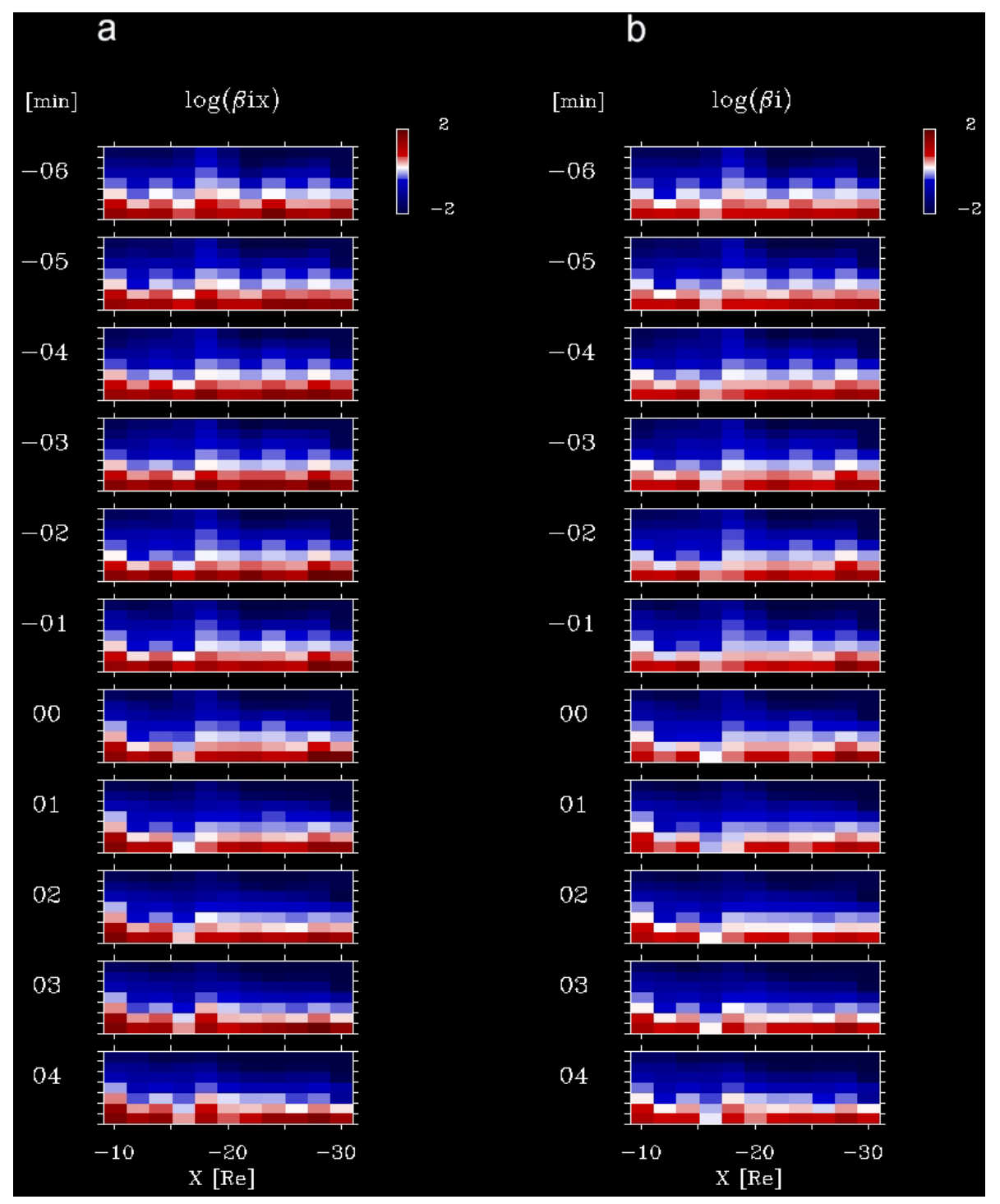

Fig. A1. Result of the analysis applied to the parameters (a) $\beta_{i x}$ and (b) $\beta_{i}$.

these are the variations found to start before dipolarization and magnetic reconnection occur. In addition, as can be seen in the panels showing $B_{z}$ and $\Delta B_{z}$, the dipolarization, and the northward increase of $B_{z}$ associated with the earthward convective flow are separate processes (Shue et al., 2008). Meanwhile, the variations in $E_{y}, F_{\text {poyz }}$, and $B_{\mathrm{rms}}$ propagate from the earthward to the tailward side, and magnetic reconnection starts when these variations reach $X \sim-20 R_{E}$.

The region around $X \sim-20 R_{E}$ is the transition region from the highly stretched, highly stressed dipole field (a catapult/slingshot current sheet) to the anti-parallel Harris-type magnetic field (Harris, 1962), characterized by less stress. If the cross-tail current in the catapult current sheet is strengthened by the enhancement in the Poynting flux toward the plasma-sheet center, the catapult current sheet moves earth- ward due to the domination of the earthward $\boldsymbol{J} \times \boldsymbol{B}$ force over the tailward pressure-gradient force.

Around the edge of the stretched dipole field, the flux tubes of the catapult current sheet and the surrounding flux tubes on the lobeside move relative to each other (Fig. 5). As a result, the surrounding flux tubes move toward the plasma-sheet center to fill the rarefied region forming a very thin currentsheet located at the center of intense earthward and tailward magnetic fields which were formerly the off-equatorial lobe magnetic fields. It is entirely possible that this boundary develops into the magnetic neutral line and that magnetic reconnection starts as a consequence.

The major difference between our model and the NENL model is that magnetic reconnection is the result of catapult current-sheet relaxation; the earthward flow due to the 
catapult current-sheet relaxation process precedes the earthward and tailward flows associated with magnetic reconnection. Therefore, the relaxation of the highly stretched dipole field line is a key to understanding the substorm onset. For this reason, we call this scheme the catapult (slingshot) current-sheet relaxation model. Accordingly, it is necessary to consider a different reconnection model that starts with the Harris solution and also identifies the mechanism to merge magnetic fields at the magnetic neutral line. Anomalous resistivity is certainly possible as an extension of large magnetic-field fluctuations. We must also study the role played by electron inertia or electron-pressure stress.

The region covered by the catapult current sheet actually connects the current-disruption region around $X=-8 R_{E}$ and the magnetic neutral line around $X=-20 R_{E}$. Both locations provide significant boundary conditions in considering the behavior of the catapult current sheet. At the inner boundary, the earthward flow and the magnetic-flux transport from the catapult current sheet affects the plasma stability, and they are responsible for the development of the current disruption. In turn, the current disruption and the total pressure decrease affect the behavior of the catapult current sheet. At the outer boundary, this flow triggers magnetic reconnection, which also produces an earthward flow. In addition, flow braking around $X \sim-13 R_{E}$ and the conversion of the plasma flow into the duskward direction are key processes related to the transfer of the momentum and energy across that region, causing current disruption and dipolarization. These are also key processes in understanding the stability of the catapult current sheet. The initial total pressure decrease seems to be closely related to the convective earthward flows.

After starting magnetic reconnection around $t=0$, this process plays a significant role by separating the magnetosphere into two parts: an inner region where current disruption, particle acceleration, and M-I coupling are highly activated, and an outer region where a slow shock (Saito et al., 1995) and plasmoid (Machida et al., 1994; Ieda et al., 1998) are formed and particle acceleration takes place.

\section{Appendix A}

For clarification and to enable us to explore the relationship between the parameters displayed and the values of $\beta_{i x}=n k T_{i} /\left(B_{x}^{2} / 2 \mu_{0}\right)$ and $\beta_{i}=n k T_{i} /\left(\left(B_{x}^{2}+B_{y}^{2}+B_{z}^{2}\right) / 2 \mu_{0}\right)$, we show the results of our analysis applied to $\beta_{i x}$ and $\beta_{i}$ in Fig. A1. We confirm that the data with higher $\beta_{i x}$ or $\beta_{i}$ values are located closer to the plasma-sheet center (midplane), and that the structures in $\beta_{i x}$ and $\beta_{i}$ are almost the same, except in the region around the midplane, where the values of $B_{x}, B_{y}$, and $B_{z}$ are small and their ratio is variable, while $B_{x}$ is large and dominates in the lobe region, resulting in the same pattern in the $\beta_{i x}$ and $\beta_{i}$ plots. We also confirm that the distribution in the $X$ direction is relatively uniform, reflecting the basic structure of the magnetotail, although there are some random variations in that direction in both the $\beta_{i x}$ and $\beta_{i}$ plots.

Acknowledgements. We wish to thank S. Kokubun and T. Nagai for the Geotail MGF magnetic-field data, K. Tsuruda and H. Hayakawa for the Geotail EFD electric-field data, G. K. Parks for the Polar UVI auroral-imager data, and S. B. Mende and H. U. Frey for the IMAGE FUV auroral-imager data. We thank T. Araki, Y. Kamide, D. Fairfield, M. Fujimoto, and the members of the World Data Center for Geomagnetism, and the Solar-Planetary Electromagnetism Laboratory at Kyoto University for helpful comments and discussions.

Topical Editor I. A. Daglis thanks two anonymous referees for their help in evaluating this paper.

\section{References}

Akasofu, S.-I.: The development of the auroral substorm, Planet. Space Sci., 12, 273-282, 1964.

Akasofu, S.-I.: Physics of Magnetospheric Substorms, D. Reidel, Dordrecht-Holland, 1977.

Angelopoulos, V., Baumjohann, W., Kennel, C. F., Coroniti, F. V., Kivelson, M. G., Pellat, R., Walker, R. J., Luhr, H., and Paschmann, G.: Bursty bulk flows in the inner central plasma sheet, J. Geophys. Res., 97, 4027-4039, 1992.

Baker, D. N., Pulkkinen, T. I., Angelopoulos, V., Baumjohann, W., and McPherron, R. L.: Neutral line model of substorms: Past results and present view, J. Geophys. Res. 101, 12975-13010, 1996.

Baumjohann, W., Paschmann, G., and Luhr, H.: Characteristics of high-speed ion flows in the plasma sheet, J. Geophys. Res., 95, 3801-3809, 1990.

Birn, J. and Hesse, M.: Substorm features in MHD simulations of magnetotail dynamics, in: Magnetospheric Substorms, Geophys. Monogra. Ser., vol. 64, edited by: Kan, J. R., Potemra, T. A., Kokubun, S., and Iijima, T., p177, AGU, Washington, D.C., 1991.

Chao, J. K., Kan, J. R., Lui, A. T. Y., and Akasofu, S.-I.: A model for thinning of the plasma sheet, Planet Space Sci., 25, 703-710, 1977.

Cheng, C. Z. and Lui, A. T. Y.: Kinetic ballooning instability for substorm onset and current disruption observed by AMPTE/CCE, Geophys. Res. Lett., 25, 4091-4094, 1998.

Fujimoto, K. and Machida, S.: A generation mechanism of electrostatic waves and subsequent electron heating in the plasma sheetlobe boundary region during magnetic reconnection, J. Geophys. Res., 111, A09216, doi:10.1029/2005JA011542, 2006.

Harris, E. G.: On a plasma sheath separating regions of oppositely directed magnetic field, Il Nuovo Cimento 23, 115-121, 1962.

Hones Jr., E. W.: The magnetotail: Its generation and dissipation, in: Physics of Solar Planetary Environments, edited by: Williams, D. J., AGU, Washington, D.C., 559, 1976.

Ieda, A., Machida, S., Mukai, T., Saito, Y., Yamamoto, T., Nishida, A. Terasawa, T., and Kokubun, S.: Statistical analysis of the plasmoid evolution with Geotail observations, J. Geophys. Res., 103, 4453-4465, 1998.

Ieda, A., Fairfield, D. H., Slavin, J. A., Liou, K., Meng, C.-I., Machida, S., Miyashita, Y., Mukai, T., Saito, Y., Nosé, M., Shue, J.-H., Parks, G. K., and Fillingim, M. O.: Longitudinal asso- 
ciation between magnetotail reconnection and auroral breakup based on Geotail and Polar observations, J. Geophys. Res., 113, A08207, doi:10.1029/2008JA013127, 2008.

Kan, J. R., Zhu, L., and Akasofu, S.-I.: A theory of substorms: Onset and subsidence, J. Geophys. Res., 93, 5624-5640, 1988.

Kokubun, S., Yamamoto, T., Acuna, M. H., Hayashi, K., Shiokawa, K., and Kawano, H.: The Geotail magnetic field experiment, J. Geomagn. Geoelectr., 46, 7-21, 1994.

Lui, A. T. Y.: Current disruption in the Earth's magnetosphere: Observations and models, J. Geophys. Res., 101, 13067-13088, 1996.

Lui, A. T. Y., Meng, C.-I., and Akasofu, S.-I.: Search for the magnetic neutral line in the near-earth plasma sheet 2. Systematic study of IMP 6 magnetic field observations, J. Geophys. Res., 82, 1547-1565, 1977.

Lui, A. T. Y., Mankofsky, A., Chang, C.-L., Papadopoulos, K., and Wu, C. S.: A current disruption mechanism in the neutral sheet: A possible trigger for substorm expansions, Geophys. Res. Lett., 17, 745-748, 1990.

Lui, A. T. Y., Zheng, Y., Zhang, Y., Livi, S., Rème, H., Dunlop, M. W., Gustafsson, G., Mende, S. B., Mouikis, C., and Kistler, L. M.: Cluster observation of plasma flow reversal in the magnetotail during a substorm, Ann. Geophys., 24, 2005-2013, 2006, http://www.ann-geophys.net/24/2005/2006/.

Lyons L. R., Wang, C.-P., and Nagai, T.: Substorm onset by plasma sheet divergence, J. Geophys. Res., 108, 1427, doi:10.1029/2003JA010178, 2003.

Machida, S., Mukai, T., Saito, Y., Obara, T., Yamamoto, T., Nishida, A., Hirahara, M., Terasawa, T., and Kokubun, S.: GEOTAIL low energy particle and magnetic field observations of a plasmoid at $X_{\mathrm{GSM}} \sim-142$ Re, Geophys. Res. Lett., 21, 2995-2998, 1994.

Machida, S., Miyashita, Y., Ieda, A., Nishida, A., Mukai, T., Saito, Y., and Kokubun, S.: GEOTAIL observations of flow velocity and north-south magnetic field variations in the near and middistant tail associated with substorm onsets, Geophys. Res. Lett., 26, 635-638, 1999.

Machida, S., Ieda, A., Mukai, T., Saito, Y., and Nishida, A.: Statistical visualization of the Earth's magnetotail during substorms by means of multidimensional superposed epoch analysis with Geotail data, J. Geophys. Res. 105, 25291-25304, 2000.

Mende, S. B., Heetderks, H., Frey, H. U., Stock, J. M., Lampton, M., Geller, S. P., Abiad, R., Siegmund, O. H. W., Habraken, S., Renotte, E., Jamar, C., Rochus, P., Gerard, J. C., Sigler, R., and Lauche, H.: Far ultraviolet imaging from the IMAGE spacecraft. 3. Spectral imaging of Lyman-alpha and OI $135.6 \mathrm{~nm}$, Space Sci. Rev., 91, 287-318, 2000.

Miura, A., Ohtani, S., and Tamao, T.: Ballooning instability and structure of diamagnetic hydromagnetic waves in a model magnetosphere, J. Geophys. Res., 94, 15231-15242, 1989.

Miyashita, Y., Machida, S., Nishida, A., Mukai, T., Saito, Y., and Kokubun, S.: GEOTAIL observations of total pressure and electric field variations in the near and mid-distant tail associated with substorm onsets, Geophys. Res. Lett. 26, 639-642, 1999.

Miyashita, Y., Machida, S., Mukai, T., Saito, Y., Tsuruda, K., Hayakawa, H., and Sutcliffe, P. R.: A statistical study of variations in the near and middistant magnetotail associated with substorm onsets: GEOTAIL observations, J. Geophys. Res., 105, 15913-15930, 2000.

Miyashita, Y., Machida, S., Liou, K., Mukai, T., Saito, Y.,
Hayakawa, H., Meng, C.-I., and Parks, G. K.: Evolution of the magnetotail associated with substorm auroral breakups, J. Geophys. Res., 108, 1353, doi:10.1029/2003JA009939, 2003.

Miyashita, Y., Machida, S., Kamide, Y., Liou, K., Fujimoto, M., Saito, M. H., Ieda, A., Nagata, D., Ressell, C. T., Christon, S. P., Nose, M., Singer, H. J., Frey,, H. U., Shinohara, I., Mukai, T., Saito, Y., and Hayakawa, H.: A state-of-the-art picture of substorm-associated evolution of the near-Earth magnetotail obtained from superposed epoch analysis, J. Geophys. Res., 114, A01211, doi:10.1029/2008JA013225, 2009..

Mukai, T., Machida, S., Saito, Y., Hirahara, M., Terasawa, T., Kaya, N., Obara, T., Ejiri, M., and Nishida, A.: The Low Energy Particle (LEP) experiment onboard the Geotail satellite, J. Geomagn. Geoelectr. 46, 669-692, 1994.

Nagai, T.: Observed magnetic substorm signatures at synchronous altitude, J. Geophys. Res., 87, 4405-4417, 1982.

Nagai, T., Fujimoto, M., Saito, Y., Machida, S., Terasawa, T., Nakamura, R., Yamamoto, T., Mukai, T., Nishida, A., and Kokubun S.: Structure and dynamics of magnetic reconnection for substorm onsets with Geotail observations, J. Geophys. Res., 103, 4419-4440, 1998.

Nakabayashi, J. and Machida, S.: Electromagnetic hybrid-code simulation of magnetic reconnection: velocity distribution functions of accelerated ions, Geophys. Res. Lett., 24, 1339-1342, 1997.

Nishida, A.: Geomagnetic Diagnosis of the Magnetosphere, Springer, Heidelberg, 1978.

Paterson, W., Frank, L., Kokubun, S., and Yamamoto, T.: Geotail survey of ion flow in the plasma sheet: Observations between 10 and 50 RE, J. Geophys Res., 103, 11811-11825, 1998.

Pu, Z. Y., Kang, K. B., Korth, A., Fu, S. Y., Zong, Q. G., Chen, Z. X., Hong, M. H., Lui, Z. X., Mouikis, C. G., Friedel, R. W. H., and Pulkkinen, T.: Ballooning instability in the presence of a plasma flow: A synthesis of tail reconnection and current disruption models for the initiation of substorms, J. Geophys. Res., 104, 10235-10248, 1999.

Rostoker, G. and Eastman, T.: A boundary layer model for magnetospheric substorms, J. Geophys. Res., 92, 12187-12201, 1987.

Roux, A., Perraut, S., Robert, P., Morane, A., Pedersen, A., Korth, A., Kremser, G., Aparicio, B., Rodgers, D., and Pellinen, R.: Plasma sheet instability related to the westward traveling surge, J. Geophys. Res., 96, 17679-17714, 1991.

Russell, C. T. and McPherron, R. L.: The magnetotail and substorms, Space Sci. Rev., 15, 205-266, 1973.

Shiokawa, K., Baumjohann, W., and Haerendel, G.: Braking of high-speed flows in the near-Earth tail, Geophys. Res. Lett., 24, 1179-1182, 1997.

Shue, J.-H., Ieda, A., Lui, A. T. Y., Parks, G. K., Mukai, T., and Ohtani, S.: Two classes of earthward fast flows in the plasma sheet, J. Geophys. Res., 113, A02205, doi:10.1029/2007JA012456, 2008.

Saito, Y., Mukai, T., Terasawa, T., Nishida, A., Machida, S., Hirahara, M., Maezawa, K., Kokubun, S., and Yamamoto, T.: Slowmode shocks in the magnetotail, J. Geophys. Res., 100, 23567 23581, 1995.

Slavin, J. A., Fairfield, D. H., Lepping, R. P., Azabo, S, Reiner, M. J., Kaiser, M., Owen, C. J., Phan, T., Lin, R., Kokubun, S., Mukai, T., Yamamoto, T., Singer, H. J., Romanov, S. A., Buechner, J., Iyemori, T., and Rostoker, G.: WIND, GEOTAIL, and 
GEOS 9 observations of magnetic field dipolarization and bursty bulk flows in the near-tail, Geophys. Res. Lett., 24, 971-974, 1997.

Smith, R. A., Goertz, C. K., and Grossmann, W.: Thermal catastrophe in the plasma sheet boundary layer, Geophys. Res. Lett., 13, 1380-1383, 1986.

Taguchi, S., Slavin, J., Kiyohara, M., Nose, M., Reeves, G., and Lepping, R.: Temporal relationship between midtail traveling compression regions and substorm onset: Evidence for nearEarth neutral line formation in the late growth phase, J. Geophys. Res., 103, 26607-26612, 1998.
Torr, M. R., Torr, D. G., Zukic, M., Johnson, R. B., Ajello, J., Banks, P., Clark, K., Cole, K., Parks, G., Tsurutani, B., and Spann, J.: A far-ultraviolet imager for international solarterrestrial physics mission, Space Sci. Rev. 71, 329-383, 1995.

Tsuruda, K., Hayakawa, H., Nakamura, M., Okada, T., Matsuoka, A., Mozer, F. S., and Schmidt R.: Electric field measurements on the GEOTAIL satellite, J. Geomagn. Geoelectr., 46, 639-711, 1994.

Tsyganenko, N. A. and Fairfield, D. H.: Global shape of the magnetotail current sheet as derived from Geotail and Polar data, J. Geophys. Res., 109, A03218, doi:10.1029/2003JA010062, 2004. 